\title{
DOKUMENTATIONEN
}

\section{Hintergrund, Ziele und Methoden der gemeinsamen Evaluation des Gesetzes über den Bundesfreiwilligendienst (BFDG) und des Gesetzes zur Förderung von Jugendfrei- willigendiensten (JFDG)}

\author{
Susanne Huth \\ INBAS-Sozialforschung GmbH| Geschäftsführerin \\ susanne.huth@inbas-sozialforschung.de
}

\section{Hintergrund}

Die Aussetzung der Wehrpflicht und damit des Zivildienstes zum 1. Juli 2011 wurde von der Bundesregierung als Chance begriffen, freiwilliges Engagement in Deutschland auf eine breitere Basis zu stellen: Mit dem neuen Bundesfreiwilligendienst (BFD), der sich auch an über 27-Jährige richtet, und dem Ausbau der bewährten Jugendfreiwilligendienste Freiwilliges Soziales Jahr (FSJ) und Freiwilliges Ökologisches Jahr (FÖJ) wurde mit über 85.000 Freiwilligen inzwischen ein historischer Höchststand in Deutschland erreicht. Zur zielgerichteten Weiterentwicklung des BFD und der Jugendfreiwilligendienste FSJ und FÖJ wird seit Mitte 2012 eine umfassende Evaluation von den Instituten INBAS-Sozialforschung, Institut für Berufliche Bildung, Arbeitsmarkt- und Sozialpolitik GmbH (INBAS) und Institut für Sozialforschung und Gesellschaftspolitik (ISG) im Auftrag des Bundesministeriums für Familie, Senioren, Frauen und Jugend (BMFSFJ) durchgeführt.

\footnotetext{
* Dieser Beitrag beruht auf einer leichten Überarbeitung der Vortragsdokumentation anlässlich des Fachtages Freiwilligendienste des Bundesministeriums für Familie, Senioren, Frauen und Jugend am 19. November 2012 in Berlin.
} 


\section{Zielsetzung und Grundlagen der Evaluation}

Beide Freiwilligendienstformate sind besondere Formen des bürgerschaftlichen Engagements und als Lern- und Bildungsdienste der Förderung der Bildungsfähigkeit von Jugendlichen bzw. dem lebenslangen Lernen verpflichtet. Vor diesem Hintergrund werden positive Wirkungen dieser Freiwilligendienste in drei Bereichen erwartet:

- Auf die Freiwilligen selbst hinsichtlich deren persönlicher und beruflicher Orientierung, dem Erwerb von individuellen Handlungskompetenzen und biografischer Langzeiteffekte (Mikroebene),

- auf die Organisationen in ihren Einsatzbereichen hinsichtlich Strukturen und Vernetzung, Personalentwicklung, Entwicklung von Einsatzbereichen, Tätigkeitsfeldern sowie sozialpädagogischer Konzepte und Anerkennungsstrukturen (Mesoebene) sowie

- auf den gesellschaftlichen Zusammenhalt im Sinne des Engagements für soziale oder ökologische Belange, der Erbringung wohlfahrtsrelevanter oder ökologischer Zusatzleistungen sowie der Förderung der Bereitschaft zum Engagement nach Beendigung des Dienstes (Makroebene).

Diese Wirkungsebenen stehen im Mittelpunkt der Evaluation und werden umfassend analysiert. Als Grundlage der Analysen auf der Wirkungsebene dienen Analysen auf der Teilnehmenden- und Strukturebene, dabei geht es bspw. um besondere Zielgruppen wie benachteiligte Jugendliche, Menschen mit Migrationshintergrund und Freiwillige der Altersgruppe ab 27 Jahren sowie die Rahmenbedingungen und Ressourcen bei den Trägern, Zentralund Einsatzstellen, die pädagogische Begleitung und Anerkennungsformen für die Freiwilligen.

Die Evaluation baut auf frühere Untersuchungen zum FÖJ (1996) und FSJ (1998), der Evaluation von FSJ und FÖJ aus den Jahren 2003 bis 2005 sowie dem Forschungsprojekt „Zivildienst als Sozialisationsinstanz für junge Männer“ aus dem Jahr 2011 auf. 
Huth, Hintergrund, Ziele und Methoden der gemeinsamen Evaluation

\section{Evaluationsbestandteile}

Die Evaluation der Freiwilligendienste FSJ/FÖJ und BFD fußt im Wesentlichen auf drei umfassenden quantitativ angelegten Befragungen der Teilnehmenden, der selbständigen Organisationseinheiten (sOE) und Träger ${ }^{1}$ sowie der Einsatzstellen. Diese Befragungen werden durch eine Kontrollgruppenbefragung, zusätzliche qualitative Elemente und weitere Forschungsansätze ergänzt und flankiert.

\subsection{Teilnehmendenbefragungen}

Die Teilnehmendenbefragung ist als quantitative Längsschnittstudie zu drei Zeitpunkten zwischen Herbst 2012 und Herbst 2014 konzipiert. D. h., dass dieselben Teilnehmenden drei Mal befragt werden, um die Ergebnisse miteinander vergleichen und die Entwicklungen der Teilnehmenden analysieren zu können. Sie soll Erkenntnisse bringen über die Lebenssituation, Kompetenzen und Motivationsstrukturen sowie über die Erfahrungen, den Kompetenzerwerb und die Perspektiven der Freiwilligen.

Die Ausgangsbefragung zu den Motivationen und Erwartungen der Freiwilligen wurde zwischen Ende August und Dezember 2012 durchgeführt. Die Zielgröße von 6000 Befragungen konnte dabei deutlich überschritten werden. Mehr als 7000 unter 27-jährige Teilnehmerinnen und Teilnehmer in FSJ, FÖJ und BFD wurden schriftlich in den Einführungsseminaren unter Anleitung von Mitarbeiterinnen und Mitarbeitern des Evaluationsteams befragt, mehr als 1000 Teilnehmende ab 27 Jahren im BFD nahmen an einer postalischen Befragung teil.

Die erste Wiederholungsbefragung zu den Erfahrungen während des Freiwilligendienstes und den Planungen im Anschluss daran wird im Juni 2013 online stattfinden, geplant sind 4000 Befragungen. Ergänzend dazu werden Teilnehmende befragt, die den Freiwilligendienst vorzeitig beendet haben.

1 Unter sOE und Träger sind die Strukturen zwischen der Zentralstelle und den Einsatzstellen zu verstehen, die u.a. mit der Teilnehmendenakquise und -auswahl sowie der pädagogischen Begleitung betraut sind. Diese Trägerdefinition wurde im Evaluationsbeirat diskutiert und einvernehmlich empfohlen. 
Die zweite Wiederholungsbefragung zu den Entwicklungen nach dem Freiwilligendienst und dessen Wirkungen ist mit einer Zielgröße von 2400 Befragungen für Herbst 2014 geplant.

Um die Ergebnisse der Längsschnittstudie zu vertiefen und zu verdichten, werden ergänzende Gruppendiskussionen mit Freiwilligendienstteilnehmenden im Frühjahr 2014 durchgeführt.

\subsection{Kontrollgruppenbefragung von „statistischen Zwillingen““}

Im Rahmen der Kontrollgruppenuntersuchung wird eine Vergleichsgruppe befragt, die der Teilnehmendengruppe so ähnlich wie möglich ist, um erheben zu können, worin die spezifischen Unterschiede und Wirkungen in Bezug auf die Freiwilligendienste liegen. Dazu werden ehemalige Klassenkameradinnen bzw. Klassenkameraden von Teilnehmenden unter 27 Jahren bzw. gleichaltrige und gleichgeschlechtliche Personen aus dem Bekanntenkreis von Teilnehmenden ab 27 Jahren befragt, die keinen Freiwilligendienst absolvieren.

Die Kontrollgruppenbefragung findet zu zwei Zeitpunkten statt, da diese Gruppe keinen einjährigen Dienst o. Ä. absolviert. Die Ausgangsbefragung zum Kenntnisstand über Freiwilligendienste und den Motivationen wurde parallel zur ersten Teilnehmendenbefragung zwischen Oktober und Dezember 2012 online durchgeführt, wobei der Zielwert von 600 bis 800 Befragungen überschritten werden konnte.

Die Wiederholungsbefragung zu Erfahrungen, Entwicklungen und Wirkungen ist für Januar 2014 mit einem Zielwert von 300 bis 400 Befragten geplant.

\subsection{Teilstandardisierte Befragungen der sOE und Träger}

Ab Ende Dezember 2012 wurden alle selbständigen Organisationseinheiten (sOE) und Träger im FSJ, FÖJ und BFD zur Teilnehmendenansprache und gewinnung für die Freiwilligendienste, zu Strukturen und Rahmenbedingungen sowie Entwicklungen und Wirkungen der Dienste befragt.

Ergänzt wird die sOE- und Trägerbefragung durch vertiefende telefonische Gespräche mit einzelnen ausgewählten sOE / Trägern und Zentralstellen (ab Mitte 2013) und durch Experteninterviews mit weiteren relevanten Akteuren wie beispielsweise Regionalbetreuerinnen und Regionalbetreuer im BFD, 
Huth, Hintergrund, Ziele und Methoden der gemeinsamen Evaluation

pädagogische Fachkräfte, Arbeitsmarktakteure oder Migrantenorganisationen (ab Herbst 2013).

\subsection{Einsatzstellenbefragung}

Eine Stichprobe von Einsatzstellen wird über eine teilstandardisierte Befragung per E-Mail mit telefonischem Nachfassen (Zielwert: 2000, ab Mitte 2013) zur Anleitung und den Erfahrungen mit den Freiwilligen, den Strukturen und Rahmenbedingungen sowie zu Entwicklungen und den Wirkungen der Freiwilligendienste innerhalb der Einsatzstelle befragt. In der Stichprobe sollen die Anteile von FSJ, FÖJ und BFD sowie die darin vertretenen Einsatzfelder abgebildet werden.

\subsection{Ermittlung von Langzeiteffekten}

Um Langzeiteffekte bei Freiwilligen und Organisationen zu untersuchen, wird ab Mitte 2014 zum einen eine Befragung von Ehemaligen durchgeführt, die vor zehn oder mehr Jahren einen Freiwilligendienst absolviert haben. Diese Personen werden durch ein so genanntes Screeningverfahren im Rahmen einer repräsentativen Bevölkerungsumfrage ermittelt. Darüber hinaus werden Schlüsselakteure, v. a. bei sOE / Trägern und Einsatzstellen, zu Langzeiteffekten in den Organisationen befragt.

\section{Ergebnisse}

Die Evaluation wird von einem Beirat begleitet und beraten, der ein- bis zweimal jährlich tagt. Besondere Einzelfragen und -ergebnisse werden im Rahmen von Workshops mit wissenschaftlichen und Praxisexpertinnen und experten vorgestellt und diskutiert.

Geplant sind zudem eine Fachtagung auf der Grundlage von Zwischenergebnissen Ende 2013 sowie eine Abschlusstagung zur Präsentation und Diskussion der Gesamtergebnisse und der Handlungsempfehlungen der Evaluation im Herbst 2015. 